\title{
Diagnosis and Treatment of Secretory Carcinoma of the Oral Cavity: Report of Two Cases and Pooled Analysis of the Recent Literature
}

Masaru Ogawa ( $\nabla$ masaru0523@gunma-u.ac.jp)

Gunma University: Gunma Daigaku https://orcid.org/0000-0001-6030-3554

\section{Satoshi Yokoo}

Gunma University: Gunma Daigaku

Takahiro Yamaguchi

Gunma University: Gunma Daigaku

Keisuke Suzuki

Gunma University: Gunma Daigaku

Mai Seki

Gunma University: Gunma Daigaku

Takahiro Shimizu

Gunma University: Gunma Daigaku

Jun Kurihara

Gunma University: Gunma Daigaku

Takaya Makiguchi

Gunma University: Gunma Daigaku

\section{Case report}

Keywords: Secretory carcinoma, Mammary analogue secretory carcinoma, ETV6-NTRK3 fusion gene, Oral cavity

Posted Date: March 4th, 2021

DOI: https://doi.org/10.21203/rs.3.rs-285629/v1

License: (c) (i) This work is licensed under a Creative Commons Attribution 4.0 International License.

Read Full License 


\section{Abstract}

Background: Secretory carcinoma (SC) is a malignancy of the salivary glands, which is similar to SC of the breast in terms of its association with neurotrophic tyrosine receptor kinase (NTRK) fusion-positive gene. SC is a recently described salivary gland tumor, and there are few reports describing oral minor salivary gland-derived SC. We reported two cases of SC in the oral cavity and reviewed the literature.

Case presentation: The patients included a 65-year-old Japanese woman presented with a mass of the upper lip and an 84-year-old Japanese man presented with a mass on the buccal mucosa, respectively. Diagnosis was based on histomorphological and immunohistochemical findings and identification of a specific translocation of the ETS variant 6-neurotrophic receptor tyrosine kinase 3 (ETV6-NTRK3) gene fusion. Case 1 was finally diagnosed using reverse transcription-polymerase chain reaction using formalin-fixed paraffin-embedded tissue samples, while case 2 was diagnosed using fluorescence in situ hybridization analysis.

Conclusion: It is highly likely for many cases of SC to be initially diagnosed as acinic cell carcinoma (AciCC) owing to their similar histological findings. The treatment strategy for minor salivary glandoriginated SC is the same as that for AciCC, but SC is often highly malignant and involves a high risk of cervical lymph node metastasis. Making an accurate diagnosis in cooperation with pathologists and confirming the presence of the ETV6-NTRK3 fusion gene using genetic analysis is important.

\section{Background}

In 1996, McDivitt et al. [1] reported mammary secretory carcinoma (SC) as a histological subtype of breast cancer. Mammary SC is caused by the ETS variant 6-neurotrophic receptor tyrosine kinase 3 (ETV6-NTRK3) fusion gene, through the phosphatidylinositol 3kinase/protein kinase B (P13K/Akt) and mitogen-activated protein kinase (MAPK) pathways [2]. In 2002, Hirokawa et al. [3] noted histological similarities between acinic cell carcinoma (AciCC) of the salivary gland and mammary SC. In 2010, Skalova et al. [4] found that the ETV6-NTRK3 fusion gene was expressed in salivary gland tumors that were previously diagnosed as AciCC and proposed the name mammary analogue SC (MASC). However, in 2017, the WHO classification of head and neck tumors described it as SC of the salivary gland [5]; hence, the name was unified to SC in this report.

SC develops in individuals in their 40s, which is a relatively early age of onset than that for AciCC; however, a childhood-onset case has also been reported [4]. SC showed no sex predilection.

Approximately $60-70 \%$ of the cases were located in the parotid gland, and the total number of cases in the major salivary glands, including the submandibular gland, accounted for approximately $70-80 \%[6$, 7]. Overall, there are few reports describing minor salivary gland-derived SCs [8-10]. The true frequency of occurrence is unclear because SC is a recently described disease entity, and it is possible that a few SC cases were previously diagnosed as AciCC. As SC is reported to be highly malignant compared to AciCC [11], differentiation between these carcinomas is important. 
We reported two cases of SC in the oral cavity and discussed the grade of malignancy of SC with pooled analysis of the recent literature.

\section{Case Presentation}

\section{Case 1}

A 65-year-old Japanese woman with a two-year history of a gradually enlarging mass on the left side of the upper lip consulted with the Department of Oral and Maxillofacial Surgery, Gunma University Hospital. On clinical examination, a painless, elastic-hard, protruding mass measuring $15 \times 10 \mathrm{~mm}$ was noted on the left side of the upper lip (Fig. 1a). The overlying mucosa was a flat surface, and the color was normal with no adhesion to the mass. The mass exhibited moderate intensity on contrast T1-weighted magnetic resonance imaging (MRI) and high intensity on short T1 inversion recovery Additionally, the continuity of the orbicularis oris muscle was retained (Fig. 1b, c). On fluorodeoxyglucose-positron emission tomography (FDG-PET), no abnormal accumulation was noted in the cervical lymph nodes, or distant organs. These clinical and MRI findings were suggestive of a benign salivary gland tumor. An excisional biopsy was performed, and as the mass was not adherent to the surrounding tissues, dissection was easy.

Macroscopic observation of the cut surface of the excisional biopsy specimen revealed that the mass was spherical and solid, and the boundary with the overlying mucosa was clear. Histopathological examination revealed that the mass was a 15-mm nodular tumor and its boundary with the surrounding tissue was clear with no evidence of encapsulation (Fig. 2a). It showed mixed characteristics of microcystic (Fig. 2b), papillary-cystic (Fig. 2c), and follicular (Fig. 2d) patterns of tumor cell proliferation. Polymorphous low-grade adenocarcinoma, AciCC, and SC were considered in the differential diagnosis based on the results of hematoxylin and eosin (HE) staining; immunostaining and special staining were performed for differentiation (Table 1).

Immunohistochemistry showed that the tumor was positive for cytokeratin 19 (CK19), S-100, vimentin, mammagloblin, gross cystic disease fluid protein 15 (GCDFP15), and GATA3. These findings are consistent with the immunostaining findings frequently observed in SC [4, 12-15]. The MIB-1 index, which is indicative of tumor cell proliferative activity, was 3\% (Fig. 3a-h). In addition, there were few periodic acid-Schiff (PAS)-positive granules in the cytoplasm of tumor cells (Fig. 4a). PAS with diastase digestion (PAS-D) staining was positive in the abundant eosinophilic homogeneous secretions in microcystic and follicular spaces (Fig. 4b). Furthermore, the examination for ETV6-NTRK3 gene fusion was performed using a formalin-fixed paraffin-embedded tissue sample, and a positive result was obtained in reverse transcription-polymerase chain reaction (RT-PCR) (Fig. 5a). Direct sequencing of the amplified RT-PCR product confirmed the presence of ETV6-NTRK3 rearrangement (Fig. 5b). These findings led to the definitive diagnosis of SC.

To ensure a malignant negative margin, additional resection was performed under general anesthesia, and the resection margin was set at $10 \mathrm{~mm}$ from the scar of the previous excisional biopsy. No residual 
tumor tissue was observed in the resected specimen. The tumor was staged pT1 cN0. Adjuvant therapy was not indicated. For five years postoperatively, the patient showed no evidence of recurrence or metastasis.

\section{Case 2}

An 84-year-old Japanese man presenting with a mass on the left buccal mucosa consulted with the Department of Oral and Maxillofacial Surgery, Gunma University Hospital. On clinical examination, an elastic-hard mass measuring $17 \times 15 \mathrm{~mm}$ was observed on the left buccal mucosa (Fig. 6a). The mass exhibited moderate intensity on contrast T1-weighted MRI, and advancement to the buccinator muscle was noted (Fig. 6b). The FDG-PET scan revealed that the maximum standardized uptake value (SUVmax) of FDG was 4.6 in the left buccal mucosa (Fig. 6c). There was no evidence of metastasis in the cervical lymph nodes or distant organs. In the biopsy specimen, microcystic and papillary-cystic patterns of tumor cells were observed that were suggestive of AciCC or SC. These clinical, histological, and MRI findings were suggestive of a malignant left buccal mucosal salivary gland tumor. Tumor resection with a 10-mm safety margin was performed under general anesthesia.

Macroscopic observation of the cut surface of the surgical specimen revealed that it was white and solid, and the boundary with the surrounding tissues was clear. Histopathological examination showed that it was a 15-mm nodular tumor, and its boundary with the surrounding tissues was clear, with no evidence of encapsulation (Fig. 7a). Furthermore, it showed mixed features of microcystic and papillary-cystic patterns of tumor cell proliferation (Fig. 7b-d).

Immunohistochemistry showed that the tumor was positive for CK19, S-100, vimentin, mammagloblin, GCDFP15, and MUC4. These findings are consistent with the immunostaining findings which are frequently observed in SC $[4,12,16]$ (Fig. 8a-i). The MIB-1 index was 10\%. In addition, genetic analysis was performed using the fluorescence in situ hybridization analysis, in which the ETV6-NTRK3 fusion gene accompanied by chromosomal translocation $\mathrm{t}(12 ; 15)(\mathrm{p} 13$; q25) was detected (Fig. 9a-d). Based on these findings, a definitive diagnosis of SC was established. The tumor was staged pT1 cN0. Adjuvant therapy was not indicated. At two years after surgery, the patient had a good prognosis with no recurrence or metastasis.

\section{Discussion}

Sixty-two cases of minor salivary gland-originated SC have been reported between 2010 and 2017 [4, 7$11,17-40]$. These cases were identified in a literature search carried out using keywords such as 'MASC', 'secretory carcinoma', 'oral cavity', 'buccal mucosa', 'lip', 'palate', 'gingiva', and 'tongue' in the PubMed and Japan Medical Abstracts Society databases. Age, sex, location in the oral cavity, size, TNM classification, treatment, metastasis, local recurrence, follow-up period, and survival rate were all described in 19 of the 62 cases. Pooled analysis of 21 cases, which included the two patients of the present case report, was performed (Table 2). Of the 21 patients, SC developed in the lips in 9 patients and in the buccal mucosa in 6 patients, including our patients. These two locations accounted for $70 \%$ of all cases. As the labial 
mucosa is classified as buccal mucosa in the oral cavity category of Union for International Cancer Control classification, the buccal mucosa accounts for approximately $70 \%$ of all cases.

Histopathologically, tumor cells in SC proliferate in microcystic, papillary-cystic, and follicular patterns [4, 6]. However, as this histological morphology is similar to that of AciCC, differentiation between SC and AciCC is difficult by using HE staining alone. Bishop et al. reported that $19 \%$ of parotid gland AciCC cases were SC [7]. Similarly, 6 of the 21 cases with oral minor salivary gland-originated SC were initially diagnosed as AciCC, suggesting that the differentiation between SC and AciCC is difficult. In our cases, immunostaining revealed that the tumor was positive for CK19, S-100, vimentin, mammaglobin, GCDFP15, GATA3, and MUC4. These markers have been reported to be useful for differentiating SC from other salivary gland tumors $[4,12-16]$. The results were consistent with the findings frequently observed in the previously reported cases of SC, aiding in differentiation (Tables 1 and 3). However, these immunohistochemical findings are not uniform in all SC and AciCC cases; therefore, it is essential to confirm the presence of the ETV6-NTRK3 fusion gene by genetic analysis to make a definite diagnosis [4, $18,37]$. Thus, an accurate diagnosis of SC can be established by the sequential use of HE histological screening followed by immunohistological investigation and genetic analysis.

Surgical resection was performed as the initial treatment in all 21 patients. Local recurrence was noted in three cases, which may have been due to surgical margin positivity in two cases and a close margin in one case. As the histopathological findings of excisional biopsy revealed a close margin in case 1 , additional resection was performed with a $10-\mathrm{mm}$ safety margin to prevent local recurrence. Although SC is considered a low-grade malignancy, additional resection should be performed to secure a safety surgical margin in positive cases and cases with a margin close to the tumor to prevent local recurrence.

Fifteen of the 21 cases with oral minor salivary gland-originated SC were treated at CT1N0, that is, in the early stage. Late cervical lymph node metastasis to cervical lymph nodes developed in three cases $(14.3 \%)$ and seven years after surgery in one case. The frequency of cervical lymph node metastasis is higher in SC than in AciCC: 8-11\% in AciCC [41] and approximately 25\% in SC [6, 21, 22, 40, 42, 43]. Sethi et al. [6] reported that many cases of intercalated duct-type cell-predominant AciCC are metastatic, and these are highly likely to be SC, thereby confirming that the frequency of cervical metastasis may be higher in SC than that in AciCC. Therefore, long-term post-operative observation may be necessary for SC cases considering the possibility of late cervical lymph nodes metastasis. In general, SC is considered as a low-grade malignancy and the treatment outcome is favorable [11]. This was supported by the fact that all 21 patients with oral minor salivary gland-originated SC survived for 4 months to 9 years. However, few patients with parotid gland-originated SC developed distant metastasis and died, suggesting a slightly poor outcome, and cases of high-grade transformation containing a highly malignant tumor component with poor outcomes have also been reported [22]. Furthermore, the possibility of differences in the disease-free survival time among AciCC cases has been previously suggested [11]. Therefore, differentiation between the two carcinoma types is important. To evaluate true malignancy and treatment outcomes of oral minor salivary gland-originated SC and AciCC, re-investigation of the cases diagnosed as AciCC in the past may be necessary. 
SC is considered as an NTRK fusion-positive cancer, together with SC of the breast and infantile fibrosarcoma [44]. When the normal NTRK gene is fused with another gene to form an NTRK fusion gene, the tropomyosin receptor kinase (TRK) fusion protein is produced, which continuously activates the phosphoinositide phospholipase $\mathrm{C} \rrbracket(\mathrm{PLC} \rrbracket), \mathrm{MAPK}$, and $\mathrm{PI} 3 \mathrm{~K}$ signal transmission pathways and promotes cancer cell proliferation $[44,45]$. Entrectinib is a potent inhibitor of TRK A, B, and C, which has been shown to have anti-tumor activity against NTRK gene fusion-positive solid tumors, including SC. The effectiveness of entrectinib was recently demonstrated in the studies of tumor alterations responsive to targeting receptor kinases-2 involving patients with NTRK fusion-positive cancer; five of the six patients with SC also responded to the treatment. Entrectinib inhibits the phosphorylation of the TRK fusion protein, which in turn inhibits its downstream signal transmission and consequently results in inhibition of the cancer cell proliferation [44-47]. Thus, it may be a useful treatment option for patients in whom surgery is not indicated and in patients with distant metastases.

\section{Conclusion}

We reported two patients with oral cavity-originated SC and performed a pooled analysis of previously reported SC cases. It is highly likely that many cases of SC were previously diagnosed as AciCC owing to their similar histological findings. The treatment strategy for minor salivary gland-originated SC is the same as that for AciCC, but SC is often highly malignant, resulting in a high risk of cervical lymph node metastasis. According to these results, making an accurate diagnosis in cooperation with pathologists and confirmation of the ETV6-NTRK3 fusion gene by genetic analysis is important.

\section{Abbreviations}

SC: Secretory carcinoma

NTRK: Neurotrophic tyrosine receptor kinase

AciCC: Acinic cell carcinoma

RT-PCR: Reverse transcription-polymerase chain reaction

P13K/Akt: Phosphatidylinositol 3-kinase/protein kinase B

MAPK: Mitogen-activated protein kinase

ETV6-NTRK3: ETS variant 6-neurotrophic receptor tyrosine kinase 3

MASC: Mammary analogue secretory carcinoma

FDG-PET: Fluorodeoxyglucose-positron emission tomography

HE: Hematoxylin and eosin 
CK19: Cytokeratin 19

GCDFP15: Gross cystic disease fluid protein 15

PAS: Periodic acid-Schiff

PAS-D: PAS with diastase digestion

PLC区: Phosphoinositide phospholipase C区

\section{Declarations}

\section{Ethics approval and consent to participate}

This study was approved by the ethics committee of Gunma University Graduate School of Medicine.

\section{Consent for publication}

Informed consent was obtained from the patients.

\section{Availability of data and materials}

All data generated or analyzed are included in this published article.

\section{Competing interests}

The authors declare that they have no competing interests.

\section{Funding}

None.

\section{Authors' contributions}

MO contributed to the conception and design, acquisition of data, analysis, and interpretation of data. TY, KS, TS,JK and TM contributed to analysis of the patient's data/findings. MS carried out the immunoassays and immunohistochemical staining. SY conceived the study, participated in its design and coordination, and helped to draft the manuscript. All authors read and approved the final manuscript.

\section{Acknowledgments}

We would like to thank Editage (www.editage.com) for English language editing.

\section{References}

1. McDivitt RW, Stewart FW. Breast carcinoma in children. JAMA 1966;195:388-90. 
2. Tognon C, Knezevich SR, Huntsman D, et al. Expression of the ETV6-NTRK3 gene fusion as a primary event in human secretory breast carcinoma. Cancer cell 2002;2:367-76.

3. Hirokawa M, Sugihara K, Sai T, et al. Secretory carcinoma of the breast: a tumour analogous to salivary gland acinic cell carcinoma? Histopathol. 2002;40:223-9.

4. Skálová A, Vanecek T, Sima R, et al. Mammary analogue secretory carcinoma of salivary glands, containing the ETV6-NTRK3 fusion gene: a hitherto undescribed salivary gland tumor entity. Am J Surg Pathol 2010;34:599-608.

5. El-Naggar AK, Chan JKC, Takata T, et al. The fourth edition of the head and neck World Health Organization blue book: editors' perspectives. Hum Pathol. 2017;66:10-12.

6. Sethi R, Kozin E, Remenschneider A, et al. Mammary analogue secretory carcinoma: update on a new diagnosis of salivary gland malignancy. Laryngoscope 2014;124:188-95.

7. Bishop,J.A. Unmasking MASK: Bringing to light the unique morphologic, immunohistochemical and genetic features of the newly recoginized mammary analogue secretory carcinoma of salivary glands. Head and Neck Pathol 2013;7:35-9.

8. Paudel D, Nishimura M, Adhikari BR, et al. Secretory Carcinoma of Minor Salivary Gland in Buccal Mucosa: A Case Report and Review of the Literature. Case Rep Pathol. 2019 Mar 27;2019:2074504. doi: 10.1155/2019/2074504. PMID: 31032132; PMCID: PMC6457284.

9. Luo W, Lindley SW, Lindley PH, et al. Mammary analog secretory carcinoma of salivary gland with high-grade histology arising in hard palate, report of a case and review of literature. Int $\mathrm{J}$ Clin Exp Pathol 2014;7:9008-22.

10. Aizawa T, Okui T, Kitagawa K, et al. A case of mammary analog secretory carcinoma of the lower lip. J Oral Maxillofac Surg Med Pathol. 2016;28:277-82.

11. Choisea S, Griffith C, Assaad A, et al. Clinicopathological characterization of mammary analogue secretory carcinoma of salivary glands. Histopathology.2012;61:387-94.

12. Mariano FV, dos Santos HT, Azañero WD, et al. Mammary analogue secretory carcinoma of salivary glands is a lipid-rich tumour, and adipophilin can be valuable in its identification. Histopathology. 2013;63:558-67.

13. Jung MJ, Song JS, Kim SY, et al. Finding and characterizing mammary analogue secretory carcinoma of the salivary gland. Korean J Pathol. 2013;47:36-43.

14. Chênevert J, Duvvuri U, Chiosea S, et al. DOG1: a novel marker of salivary acinar and intercalated duct differentiation. Mod Pathol.. 2012;25:919-29.

15. Andrade E.P.D, Teixeira L.N, Montalli V.A.M, et al. Epithelial membrane antigen and DOG1 expression in minor salivary grand tumors. Ann Diagn Pathol. 2019; doi: 10.1016/j. 2019.151408.

16. Khurram SA, Sultan-Khan J, Atkey N, et al. Cytogenetic and immunohistochemical characterization of mammary analogue secretory carcinoma of salivary glands. Oral Surg Oral Med Oral Pathol Oral Radiol. 2016;122:731-42. 
17. Kratochvil FJ III, Stewart JC, Moore SR. Mammary analog secretory carcinoma of salivary glands: a report of 2 cases in the lips. Oral Surg Oral Med Oral Pathol Oral Radiol 2012;114:630-5.

18. Griffith CC, Stelow EB, Saqi A, et al. The cytological features of mammary analogue secretory carcinoma: a series of 6 molecularly confirmed cases. Cancer Cytopathol 2013;121:234-41.

19. Laco J, Švajdler M Jr, Andrejs J, et al. Mammary analog secretory carcinoma of salivary glands: a report of 2 cases with expression of basal/myoepithelial markers (calponin, CD10 and p63 protein). Pathol Res Pract. 2013;209:167-72.

20. Helkamaa T, Rossi S, Mesimäki K, et al. Mammary analog secretory carcinoma of minor palatal salivary glands: A case report and review of the literature. 2015;27:698-702.

21. Majewska $H$, Skalova A, Stodulski $D$, et al. Mammary analogue secretory carcinoma of salivary glands: a new entity associated with ETV6 gene rearrangement. Virchows Arch 2015;466:245-54.

22. Skálová A, Vanecek T, Simpson RH, et al. Mammary Analogue Secretory Carcinoma of Salivary Glands: Molecular Analysis of 25 ETV6 Gene Rearranged Tumors With Lack of Detection of Classical ETV6-NTRK3 Fusion Transcript by Standard RT-PCR: Report of 4 Cases Harboring ETV6-X Gene Fusion. Am J Surg Pathol 2016;40:3-13.

23. Hindocha $\mathrm{N}$, Wilson $\mathrm{MH}$, Pring $\mathrm{M}$, et al. Mammary analogue secretory carcinoma of the salivary glands: a diagnostic dilemma. Br J Oral Maxillofac Surg. 2017;55:290-2.

24. Bissinger O, Götz C, Kolk A, et al. Mammary analogue secretory carcinoma of salivary glands: diagnostic pitfall with distinct immunohistochemical profile and molecular features. Rare Tumors. 2017; doi: 10.4081/rt.2017.7162.

25. Kai K., Minesaki A., Suzuki K., et al. Difficulty in the cytodiagnosis of mammary analogue secretory carcinoma: survey of 109 cytologists with a case originating from a minor salivary gland. Acta Cytologica. 2017;61:469-76.

26. Boliere C, Murphy J, Qaisi M, et al. Mammary Analogue Secretory Carcinoma of the Palate: Case Report and Review of the Literature. Case Rep Dent 2019; doi: 10.1155/2019/7416302.

27. Griffith C, Seethala R, Chiosea SI. Mammary analogue secretory carcinoma: a new twist to the diagnostic dilemma of zymogen granule poor acinic cell carcinoma. Virchows Arch 2011;459:117-8.

28. Abe M, Inaki R, Kanno $Y$, et al. Molecular analysis of a mammary analog secretory carcinoma in the upper lip: novel search for genetic and epigenetic abnormalities in MASC. Int J Surg Case Rep 2015; 9:8-11.

29. Connor A, Perez-Ordo nez B, Shago M, et al. Mammary analog secretory carcinoma of salivary gland origin with the ETV6 gene rearrangement by FISH: expanded morphologic and immunohistochemical spectrum of a recently described entity. Am J Surg Pathol 2012;36:27-34.

30. Ito $Y$, Ishibashi $K$, Masaki A, et al. Mammary analogue secretory carcinoma of salivary glands: a clinicopathologic and molecular study including 2 cases harboring ETV6-X fusion. Am J Surg Pathol 2015;39:602-10.

31. Keisling M, Bianchi M, Pascasio JM. Mammary analog secretory carcinoma of salivary gland in a 5 year old: case report. Int J Ped Otorhinolaryngol Extra 2014;9:163-5. 
32. Serrano-Arevalo ML, Mosqueda-Taylor A, Domınguez-Malagon H, Michal M. Mammary analogue secretory carcinoma (MASC) of salivary gland in four Mexican patients. Med Oral Patol Oral Cir Bucal 2015;20: e23-e29.

33. Stevens TM, Kovalovsky AO, Velosa C, et al. Mammary analog secretory carcinoma, low-grade salivary duct carcinoma, and mimickers: a comparative study. Mod Pathol 2015;28:1084-100.

34. Din NU, Fatima S, Kayani N. Mammary analogue secretory carcinoma of salivary glands: a clinicopathologic study of 11 cases. Ann Diag Pathol 2016;22:49-53.

35. Zardawi IM, Hook P. Mammary analogue secretory carcinoma of minor salivary glands. Pathology 2014;46:667-9.

36. Guilmette J., Nielsen G. P., Faquin W. C., et al. Ultrastructural characterization of mammary analogue secretory carcinoma of the salivary glands: a distinct entity from acinic cell carcinoma? Head \& Neck Pathology. 2017;11:419-26.

37. Khurram S. A., Sultan-Khan J., Atkey N., Speight P. M. Cytogenetic and immunohistochemical characterization of mammary analogue secretory carcinoma of salivary glands. Oral Surgery, Oral Medicine, Oral Pathology, Oral Radiology, and Endodontology. 2016;122:731-42.

38. Roy S, Saluja K, Zhu H, Zhao B. Mammary Analogue Secretory Carcinoma of Minor Salivary Glands: A Rare Case Series and Review of the Literature. Ann Clin Lab Sci. 2018;48:94-9.

39. Stevens T. M., Kovalovsky A. O., Velosa C., et al. Mammary analog secretory carcinoma, low-grade salivary duct carcinoma and mimickers: a comparative study. Modern Pathology. 2015;28:1084100.

40. Urano M., Nagao T., Miyabe S., et al. Characterization of mammary analogue secretory carcinoma of the salivary gland: discrimination from its mimics by the presence of the ETV6-NTRK3 translocation and novel surrogate markers. Human Pathology. 2015;46:94-103.

41. Neville BW, Damm DD, Allen CM, editors. Oral and maxillofacial pathology. 2016. 4th ed. An Imprint of Elsevier; p.457-9.

42. Chiosea SI, Griffith C, Assaad A, Seethala RR. The profile of acinic cell carcinoma after recognition of mammary analog secretory carcinoma. Am J Surg Pathol. 2012;36:343-50.

43. Shah AA, Wenig BM, LeGallo RD, et al. Morphology in conjunction with immunohistochemistry is sufficient for the diagnosis of mammary analogue secretory carcinoma. Head Neck Pathol. 2015;9:85-95.

44. Cocco E, Scaltriti M, Drilon A. NTRK fusion-positive cancers and TRK inhibitor therapy. Nat Rev Clin Oncol. 2018;15:731-47.

45. Vaishnavi A, Le AT, Doebele RC, et al. TRKing down an old oncogene in a new era of targeted therapy. Cancer Discov. 2015;5:25-34.

46. Kheder ES, Hong DS. Emerging targeted therapy for tumors with NTRK fusion proteins. Clin Cancer Res. 2018;24:5807-14. 
47. Rolfo C, Ruiz R, Giovannetti E, et al. Entrectinib: a potent new TRK, ROS1, and ALK inhibitor. Expert Opin Investig Drugs. 2015;24:1493-500.

\section{Tables}

Due to technical limitations, tables 1 to 3 PDFs are only available as a download in the Supplemental Files section.

\section{Figures}
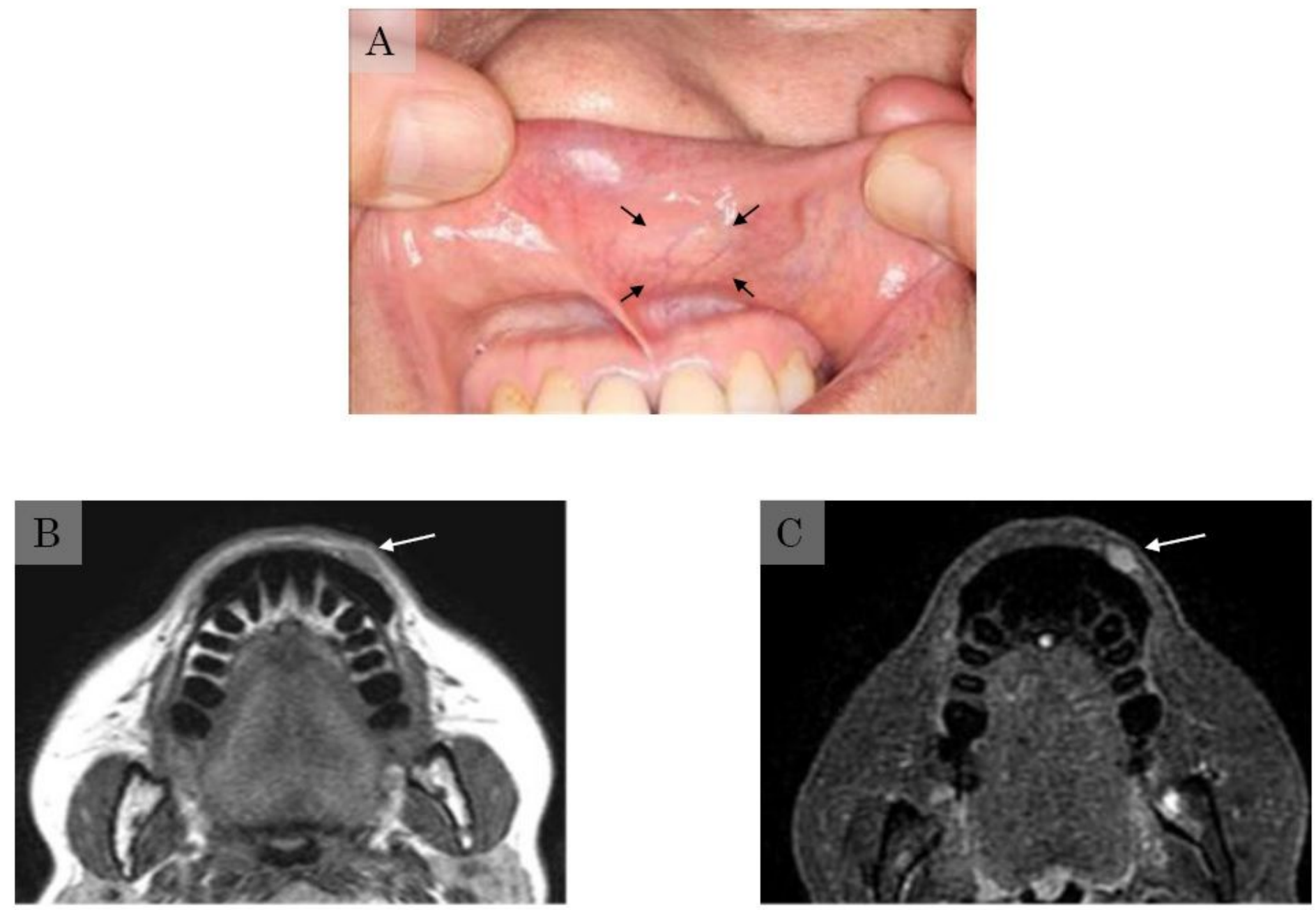

\section{Figure 1}

Clinical findings A elastic-hard protruding mass of $15 \times 10 \mathrm{~mm}$ was noted on the left side of the upper lip (A). The mass exhibited moderate intensity on contrast T1-weighted magnetic resonance imaging (B) and high intensity on short T1 inversion recovery (C). The continuity of the orbicularis oris muscle was retained. 

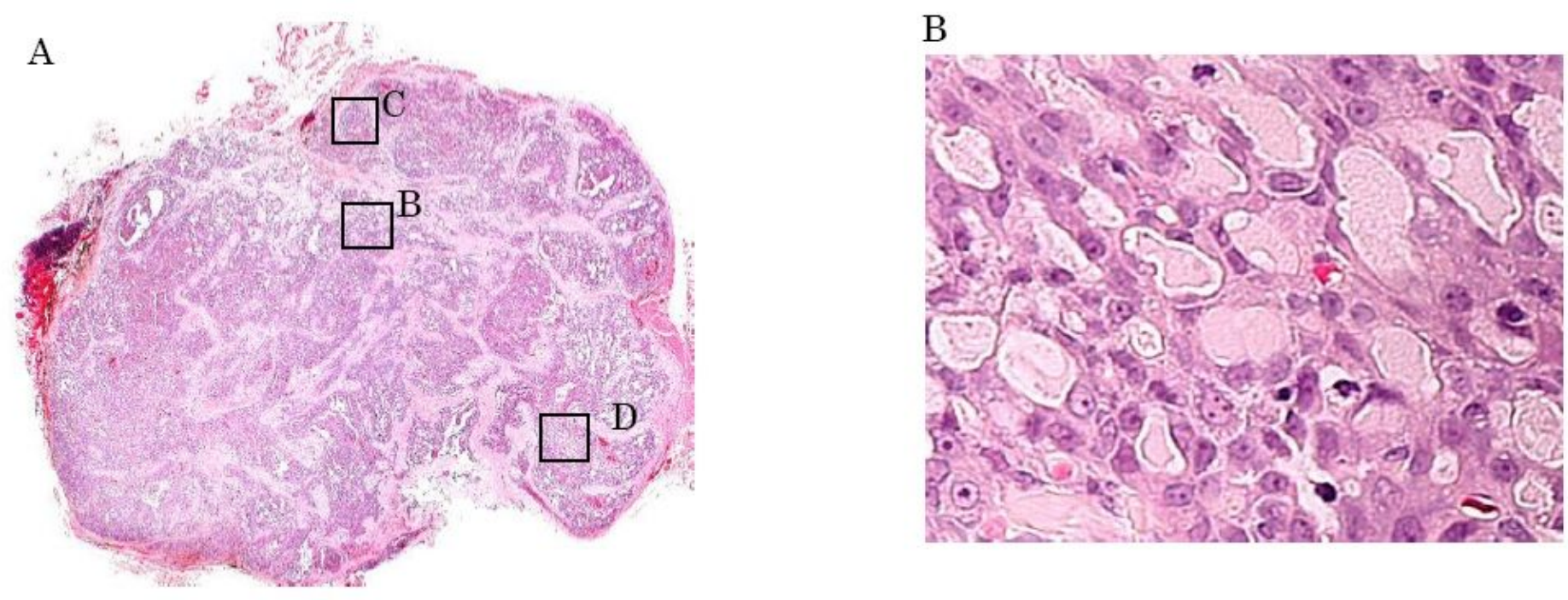

$\mathrm{C}$

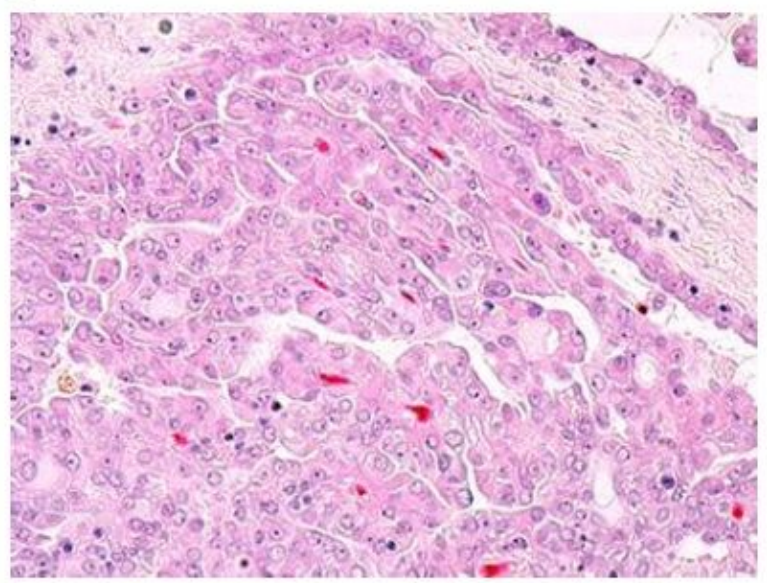

$\mathrm{D}$

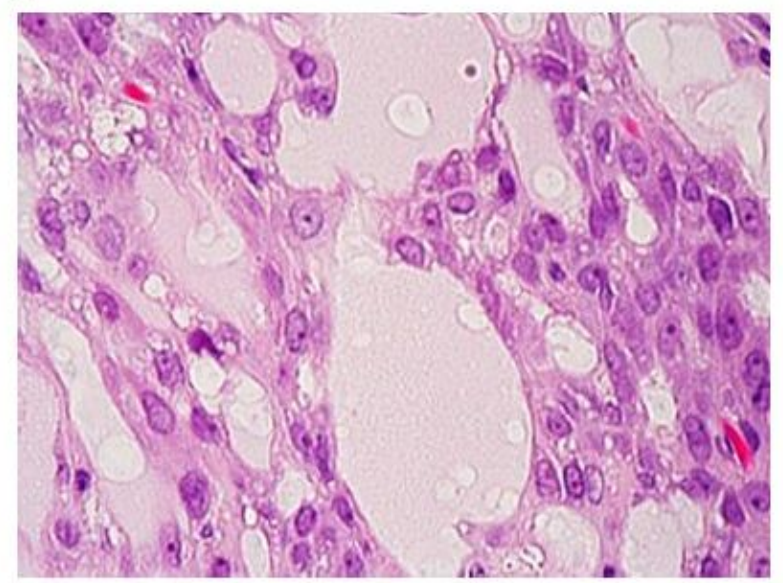

\section{Figure 2}

Histological findings (hematoxylin and eosin staining) The lesion was a nodular tumor and the boundary with the surrounding area was clear, with no evidence of encapsulation (A) (magnification 10x). Microcystic (B) (magnification 200x), papillary-cystic (C) (magnification 200x), and follicular (D) (magnification 400x) patterns of tumor cell proliferation were mixed. 
A

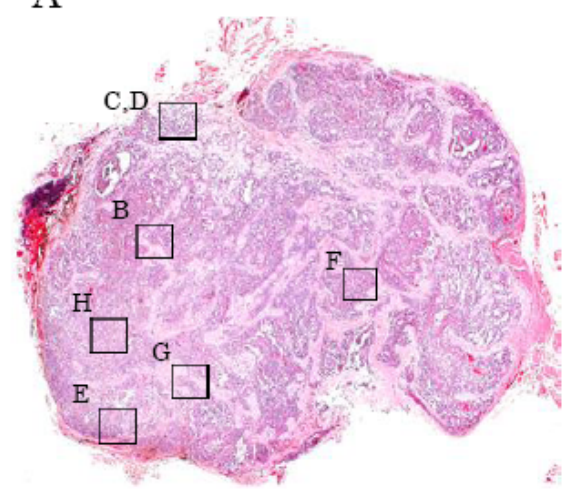

$\mathrm{D}$

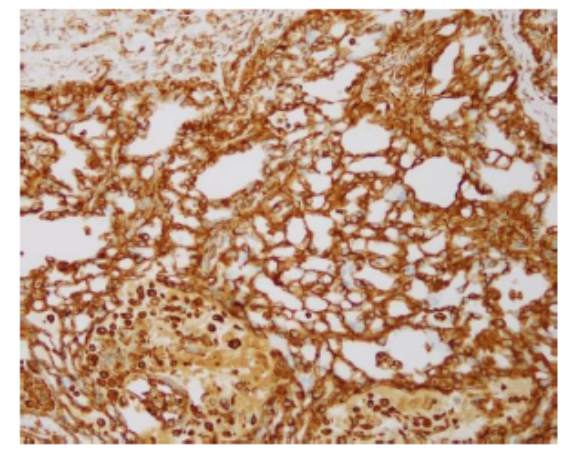

G

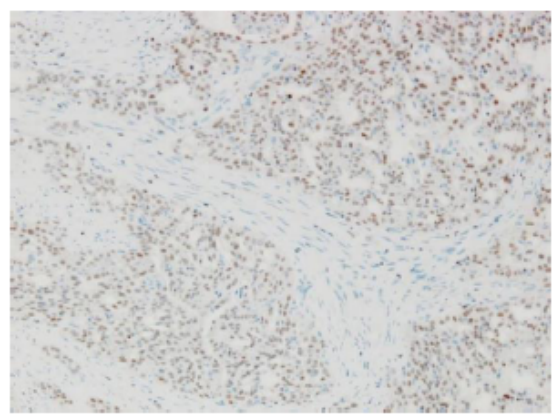

B

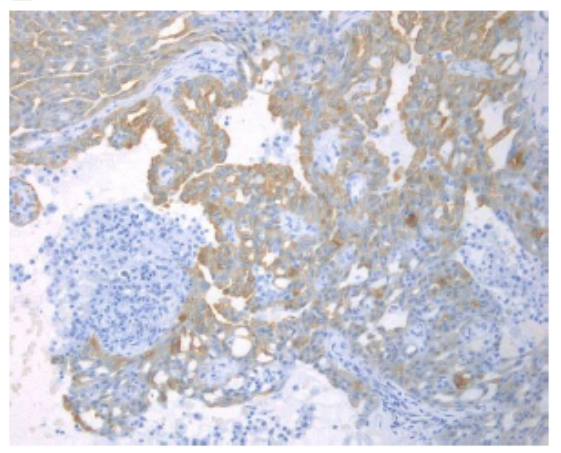

$\mathrm{E}$

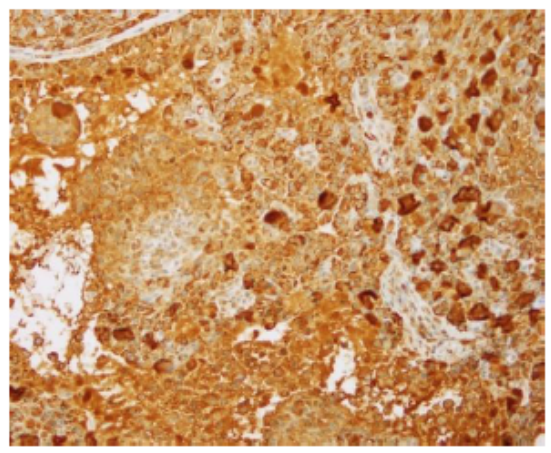

$\mathrm{H}$

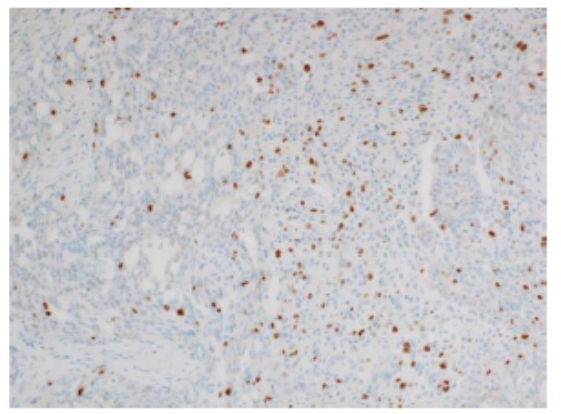

$\mathrm{C}$

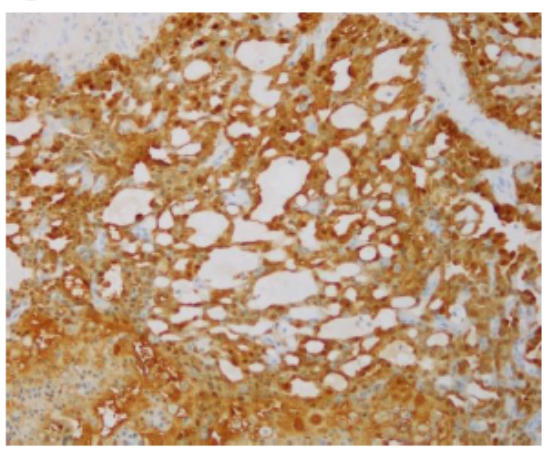

$\mathrm{F}$

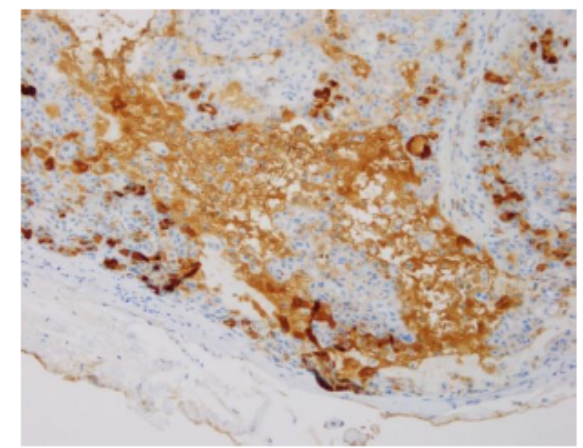

\section{Figure 3}

Immunohistochemical findings Hematoxylin and eosin (A) and immunostaining with Cytokeratin 19 (B), S-100 (C), Vimentin (D), Mammaglobin (E), gross cystic disease fluid protein 15 (F), and GATA3 (G) (magnification 100x). An index of tumor cell proliferative activity, the MIB-1 index, was 3\% (H) (magnification 100x). 


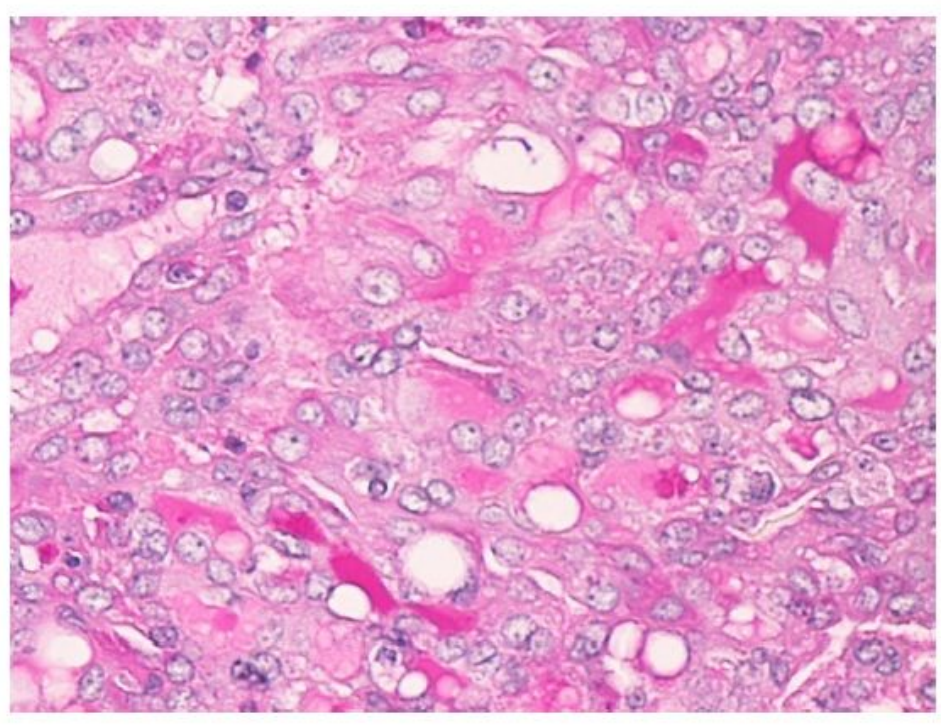

A

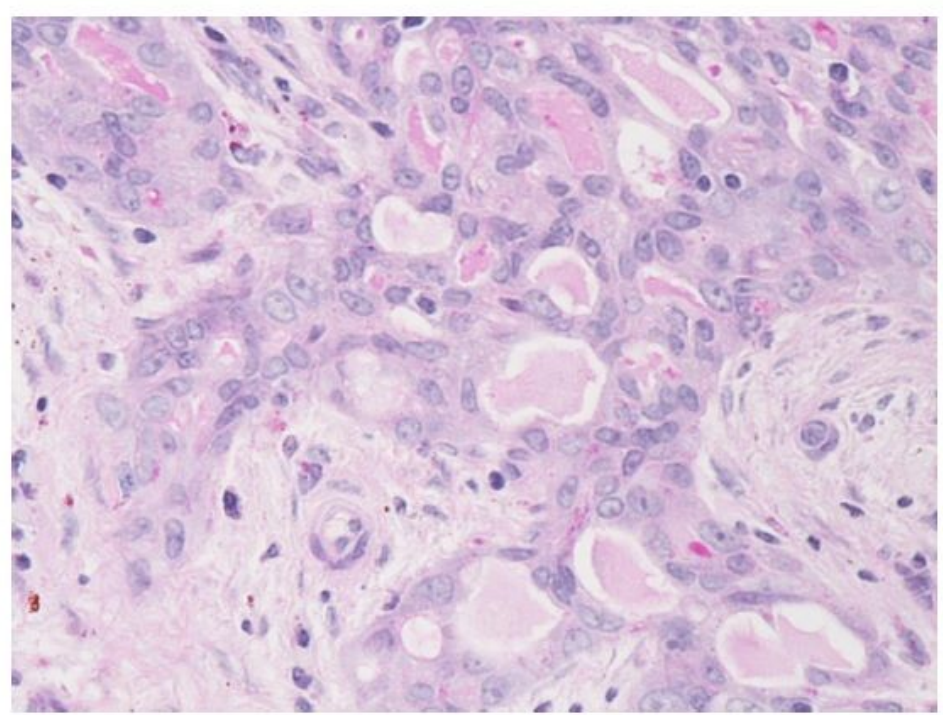

B

\section{Figure 4}

Special staining findings There were few periodic acid-Schiff (PAS)-positive granules in the tumor cell cytoplasm (A) (magnification 400x). Diastase digestion PAS staining was positive in abundant eosinophilic homogeneous secretions in microcystic and follicular spaces (B) (magnification 400x).
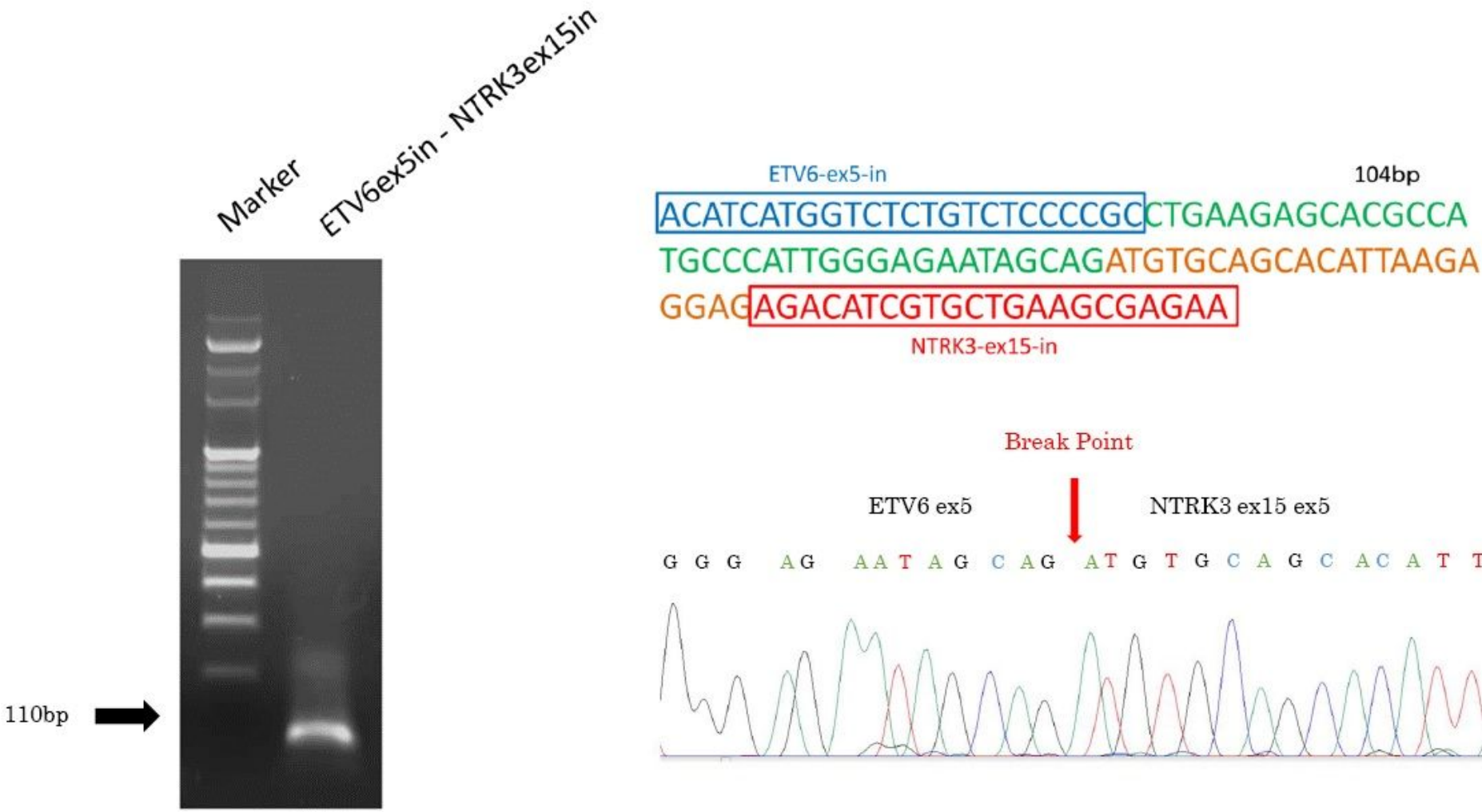

Fusion gene(104bp)

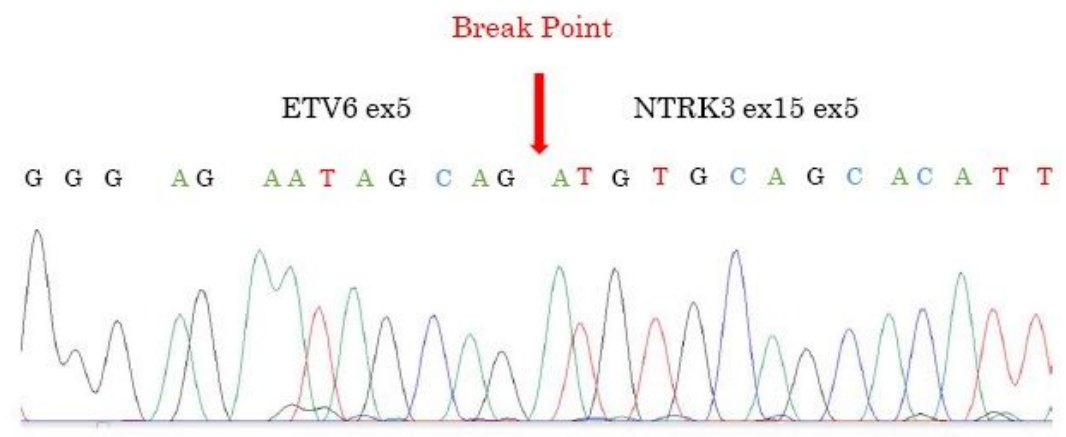




\section{Figure 5}

Reverse transcription-polymerase chain reaction (RT-PCR) for the detection of ETV6-NTRK3 fusion gene transcripts RT-PCR analysis showed amplification of the ETV6-NTRK3 fusion gene (A). Direct sequencing of the amplified RT-PCR product confirmed the presence of ETV6-NTRK3 rearrangement (B)
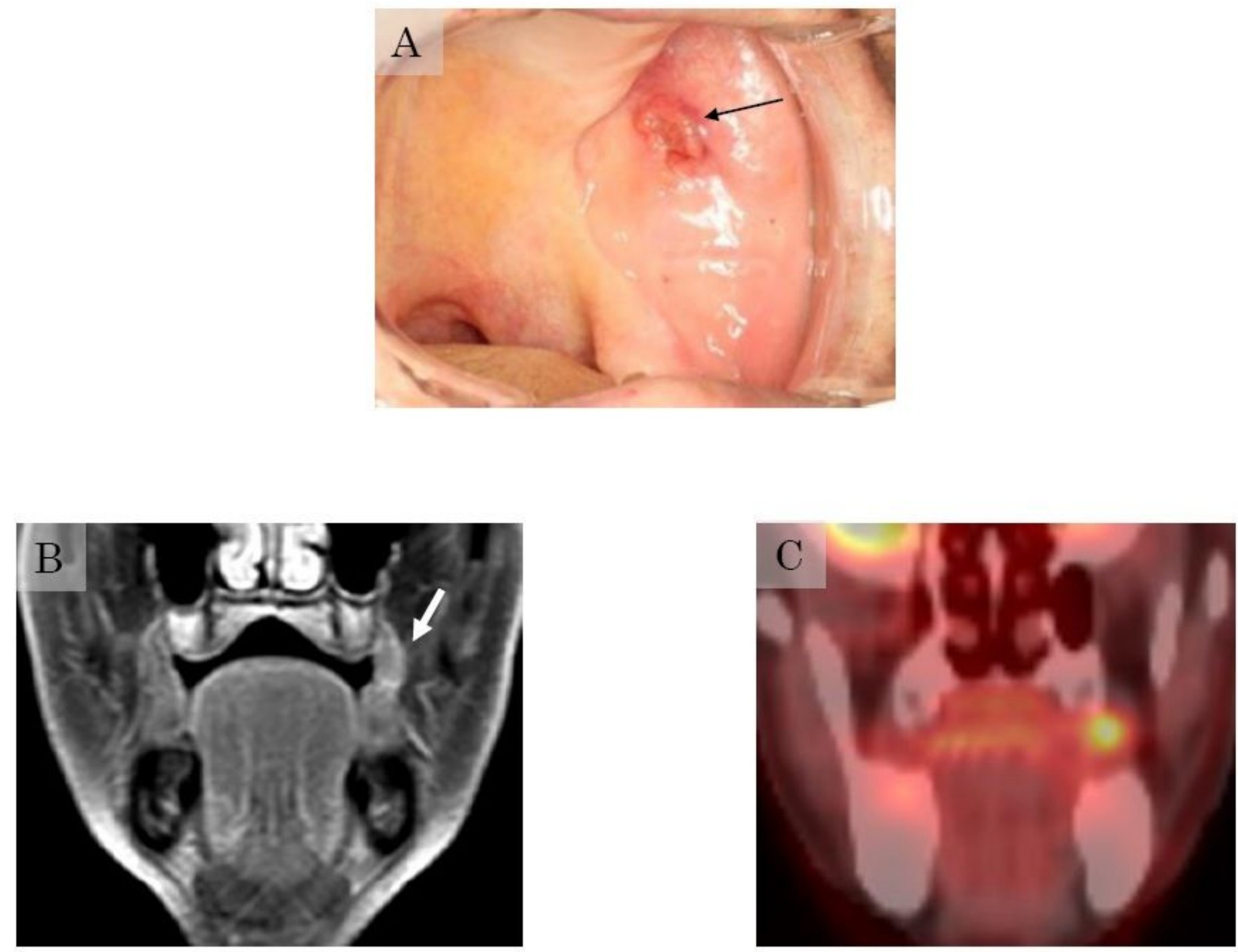

\section{Figure 6}

Clinical findings An elastic-hard mass of $17 \times 15 \mathrm{~mm}$ in size was observed on the left buccal mucosa (A). The mass exhibited moderate intensity on contrast T1-weighted magnetic resonance imaging and advancement to the buccinator muscle was noted (B). On fluorodeoxyglucose-positron emission tomography (FDG-PET) with an SUVmax of 4.56, FDG accumulation was detected in the left buccal mucosa (C). 
A

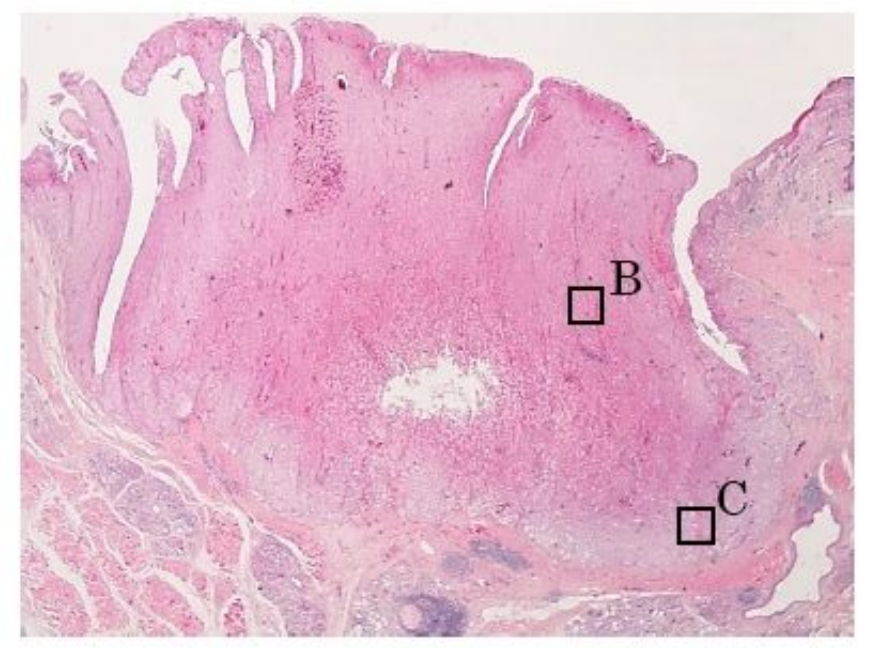

B

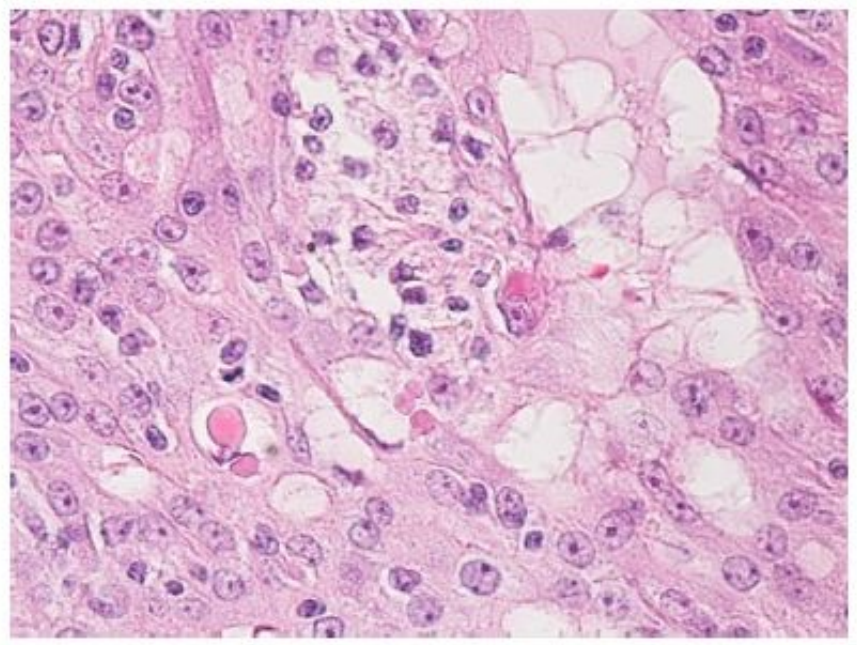

$\mathrm{C}$

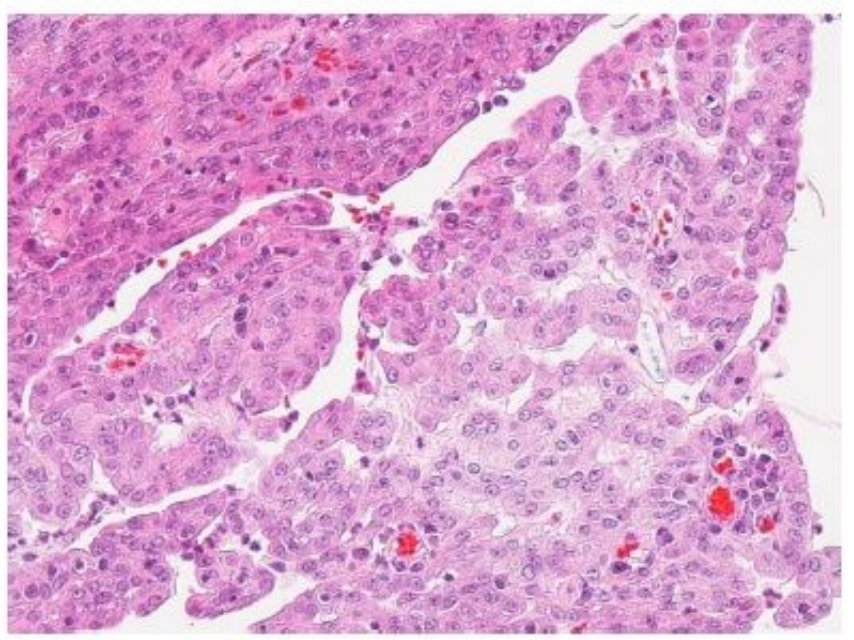

Figure 7

Histological findings (hematoxylin and eosin staining) The lesion was a nodular tumor and the boundary with the surrounding area was clear, with no evidence of encapsulation (A) (magnification 10x). Microcystic (B) (magnification 400x) and papillary-cystic (C) (magnification 200x) patterns of tumor cell proliferation were mixed. 
A

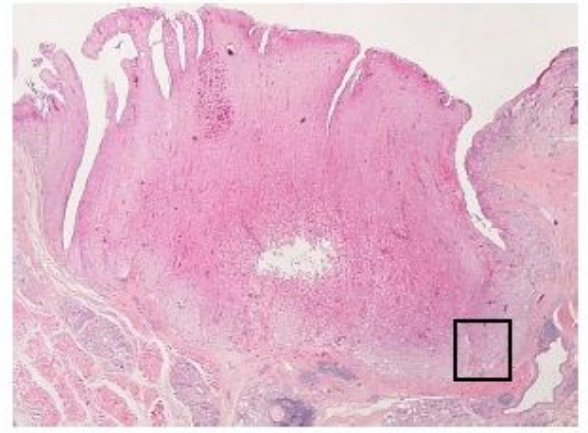

D

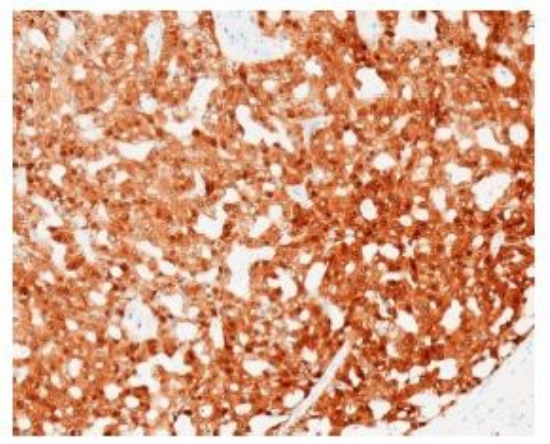

G

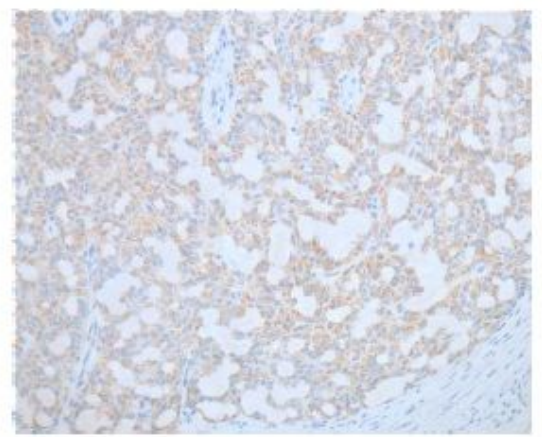

B

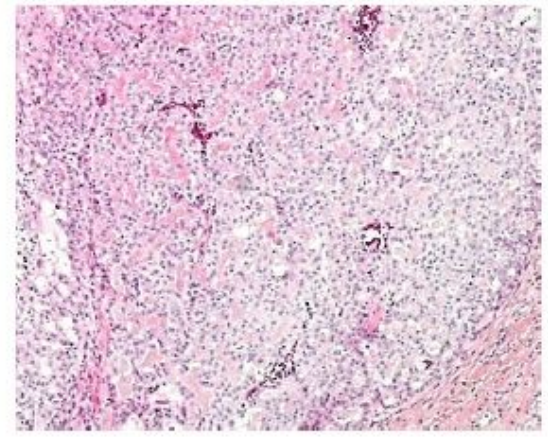

E

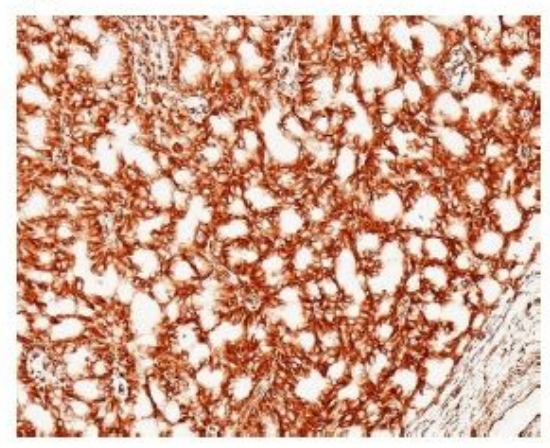

$\mathrm{H}$

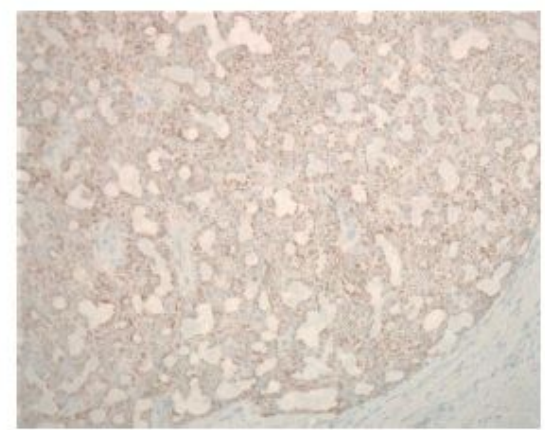

C

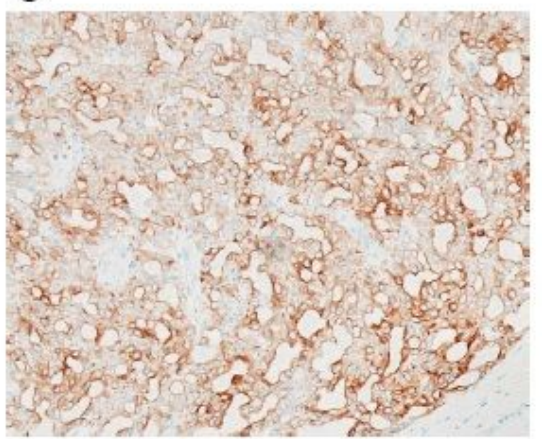

F

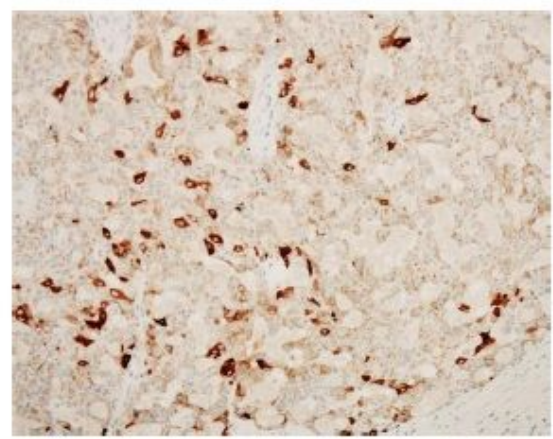

I

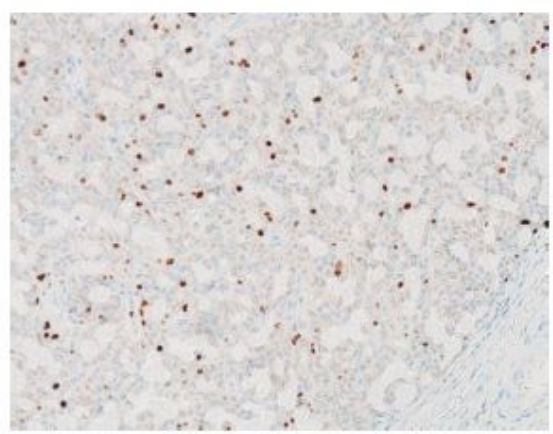

\section{Figure 8}

Immunohistochemical findings Hematoxylin and eosin (A,B) (magnification 10x) and immunostaining with Cytokeratin 19 (C), S-100 (D), Vimentin (E), Mammaglobin (F), gross cystic disease fluid protein 15 $(G)$, and MUC4 (H) (magnification 200x). An index of tumor cell proliferative activity, the MIB-1 index, was $3 \%$ (I) (magnification 100x). 


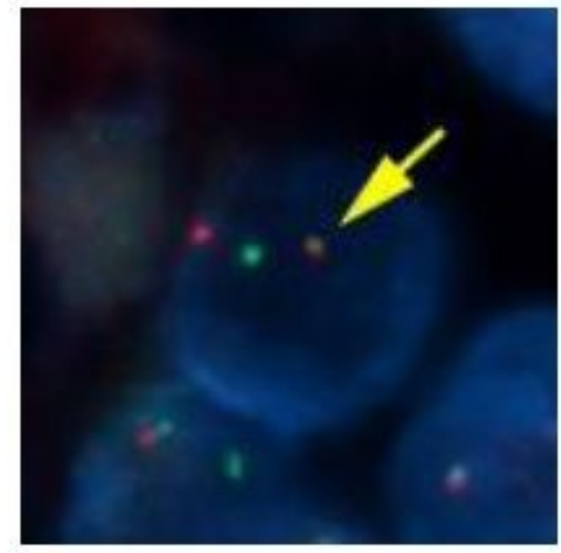

\section{(A)}

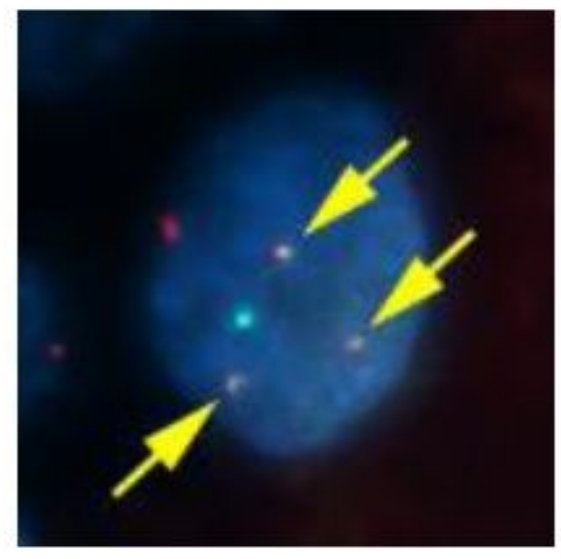

(C)

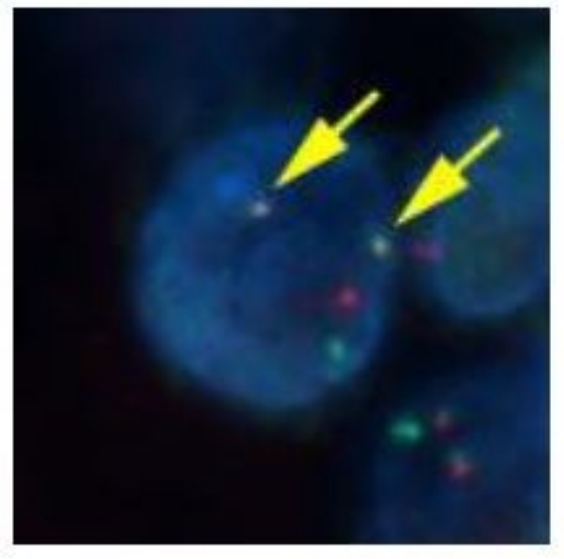

\section{(B)}

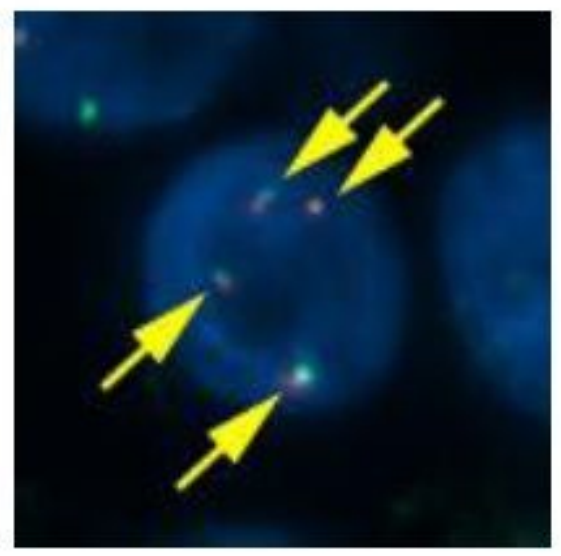

(D)

Figure 9

ETV6-NTRK3 fluorescence in situ hybridization Signals of the ETV6 and NTRK3 probes are presented in green and red, respectively. The number of fusion gene signals was classified into four patterns: 1 fusion gene signal (A), 2 signals (B), 3 signals (C), and 4 signals (D).

\section{Supplementary Files}

This is a list of supplementary files associated with this preprint. Click to download.

- Table1.pdf

- Table2.pdf

- Table3.pdf 